\title{
Surgical anatomy of the round window with special reference to cochlear implantation
} by

Burkhard K. H. Franz, Graeme M. Clark and David M. Bloom (Melbourne, Australia)

\begin{abstract}
When the multi-channel cochlear implant electrode is inserted into the scala tympani through the round window the operation is best performed via a posterior tympanotomy. The view of the round window membrane, however, is incomplete because of its orientation and the fact that it has a conical shape. Nevertheless, a good view along the basal turn is obtained after the antero-inferior overhang of the round window niche and the crista fenestrae have been removed. It might be damaging to drill away the postero-superior overhang as the osseous spiral lamina lies extremely close to the round window membrane.
\end{abstract}

\section{Introduction}

Different approaches for placing the cochlear implant electrode as close as possible to the nerve fibres have been described (Chouard and MacLeod, 1976; Clark, 1975; Kubik, 1984; Simmons, 1966). The round window as an access for inserting an electrode array has advantages. The surgical approach is relatively easy and the electrode can be placed close to the tonotopically organised auditory nerve fibres in the spiral lamina. Thus the insertion of the electrode into the scala tympani through the round window has been used by many surgeons (Burian et al., 1981; Clark et al., 1979; House and Urban, 1973; Michelson, 1971; Michelson and Schindler, 1981).

Difficulties encountered when inserting an electrode array through the round window using an endaural approach were discussed by Banfai in 1978. With this approach the electrode hit the modiolus and was deflected towards the outer wall before passing along the basal turn. To overcome this difficulty and to obtain a better view along the basal turn of the cochlea the lowest point of the basal turn was recommended for the electrode insertion, the most favourable site being $3-4 \mathrm{~mm}$. in front of the round window.

When inserting an electrode through the round window via a posterior tympanotomy the modiolus is not in the way. The electrode can be passed directly along the basal turn of the cochlea, and it can be inserted an extra few millimeters (Clark et. al., 1979). Ideally the electrode insertion via a posterior tympanotomy is performed correctly when the anteroinferior overhang of the round window niche and the crista fenestrae are removed. The postero-superior overhang of the round window niche prevents complete visibility of the window membrane. In order to obtain a better view of the window the surgeon is tempted to remove the obstructing postero-superior overhang. However, in approaching the round window in this fashion there is a risk 


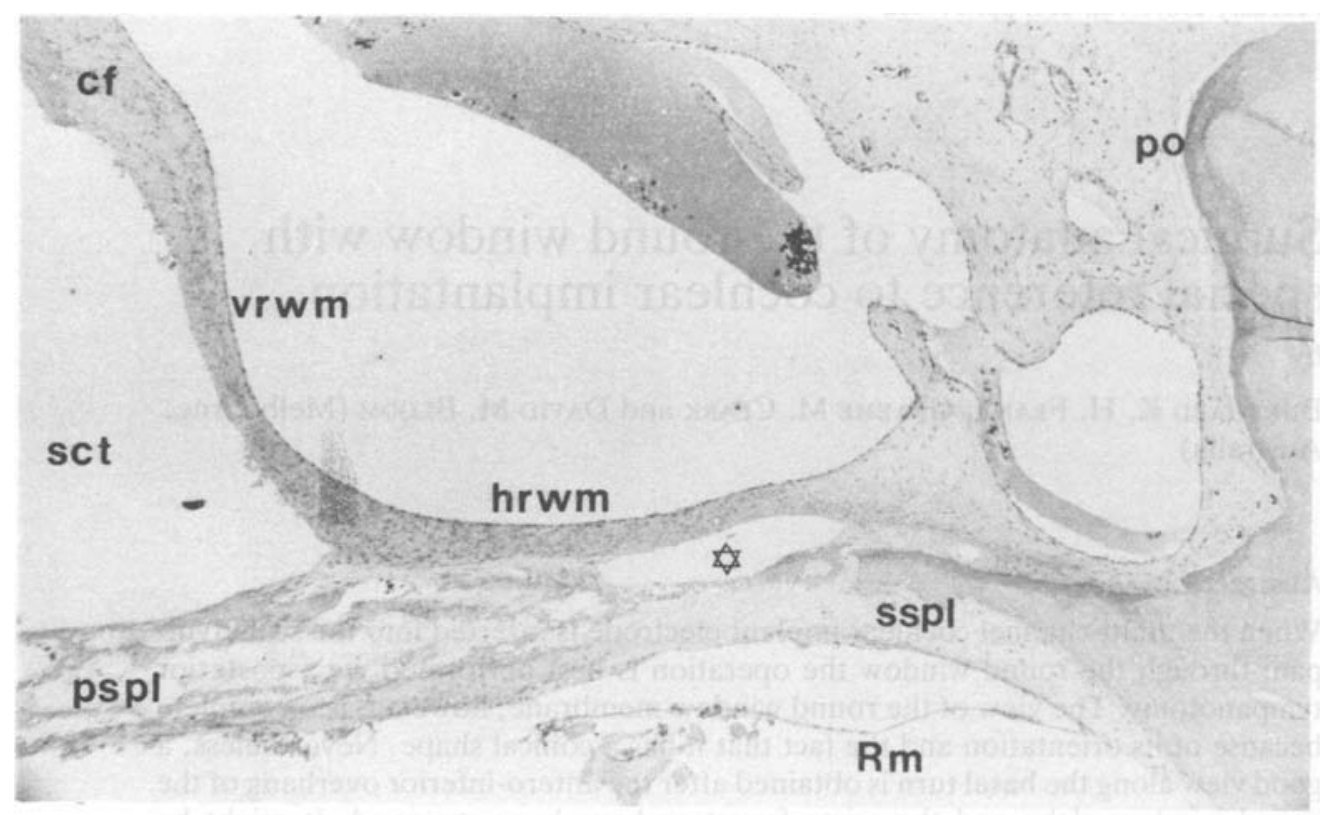

FIG. 1

Sagittal section of the right human cochlea. The anterior and posterior portions of the round window membrane form a right angle to each other. The posterior portion, hidden by the postero-superior overhang, lies very close to the osseous spiral lamina. The distance between the window membrane and the osseous spiral lamina is only $0.1 \mathrm{~mm}$. at the posterior periphery of the round window. cf: crista fenestrae; sct: scala tympani; pspl: primary spiral lamina; sspl: secondary spiral lamina; Rm: Reissner's membrane; hrwm: horizontal portion of window membrane; vrwm: vertical portion of window membrane; po: postero-superior overhang; ${ }^{*}$ blind end of scala tympani.

that the osseous spiral lamina will be traumatised.

The objective of the present study was to show the particular shape of the round window membrane, and the relationship between the round window and the osseous lamina. Furthermore we wanted to establish that the electrode inserted from a posterior tympanotomy would move along the outer wall of the basal turn once the antero-inferior overhang and the crista fenestrae had been removed.

In anatomical textbooks it can be found that the round window niche is formed by the antero-inferior and posterior bony overhang. The posterior overhang, however, does also lie superior to the round window membrane. Therefore this part of the window niche is termed the postero-superior overhang in the following text.

\section{Method}

Five temporal bones from the cat, which is our experimental animal, and five humar temporal bones were compared macroscopically. For microscopic comparison : right human temporal bone was removed 24 hours after death and fixed in 10 per cen 1 formalin, and a right cat temporal bone was removed after intra-arterial perfusion witt phosphate buffered saline containing heparir and followed by Karnovsky's fixative in phos. phate buffer. The temporal bones were decalcified in EDTA and embedded in Spurr': resin. The cat temporal bone was sectioned ir the midmodiolar plane running through the round window. The human temporal bone was sectioned in the sagittal plane. Section: were stained with haematoxylin-eosin anc viewed under a light microscope.

\section{Results}

The round window membrane of the human and of the cat has a conical shape witl 
a peak directed towards the scala tympani. The conical shape of the round window results from fusion with the base of the primary osseous spiral lamina (Fig. 1). There are three surfaces of the conical cap: two large membraneous ones that gradually form a right angle to each other; and a small bony surface that constitutes the fusion with the osseous spiral lamina. One of the membraneous surfaces of the cone lies in the horizontal plane. This portion forms the posterior part of the round window membrane and is slightly smaller than the anterior portion which lies in a vertical plane.

The conical shape of the round window resembles the tympanic membrane. However, the cone of the round window membrane has a peak that lies off-centre and thereby gives the cone an asymmetric shape. In the cat the conical shape of the round window membrane can be observed with the operating microscope as the membrane is not hidden underneath bony structures. In the human the round window membrane is difficult to see as most of it is covered by the postero-superior and antero-inferior overhang.

In approaching the round window from a posterior tympanotomy the surgeon faces the anterior portion of the membrane that lies in a vertical plane. The posterior portion of the membrane that lies in the horizontal plane cannot be seen as it is covered completely by the postero-superior overhang (Figs 1 and 2).

In the human and in the cat the horizontal portion of the round window membrane lies extremely close to the osseous spiral lamina (Figs. 1 and 3). In the human the distance between the round window membrane and the osseous spiral lamina is only $0.1 \mathrm{~mm}$. at the posterior periphery; however, the distance increases a little towards the centre of the round window to about $1 \mathrm{~mm}$.

From the posterior tympanotomy the very beginning of the outer wall of the basal turn cannot be seen unless the crista fenestrae is removed. The crista fenestrae narrows the basal turn in contrast to the gradually widening basal turn of the cochlea further in (Fig. 4).

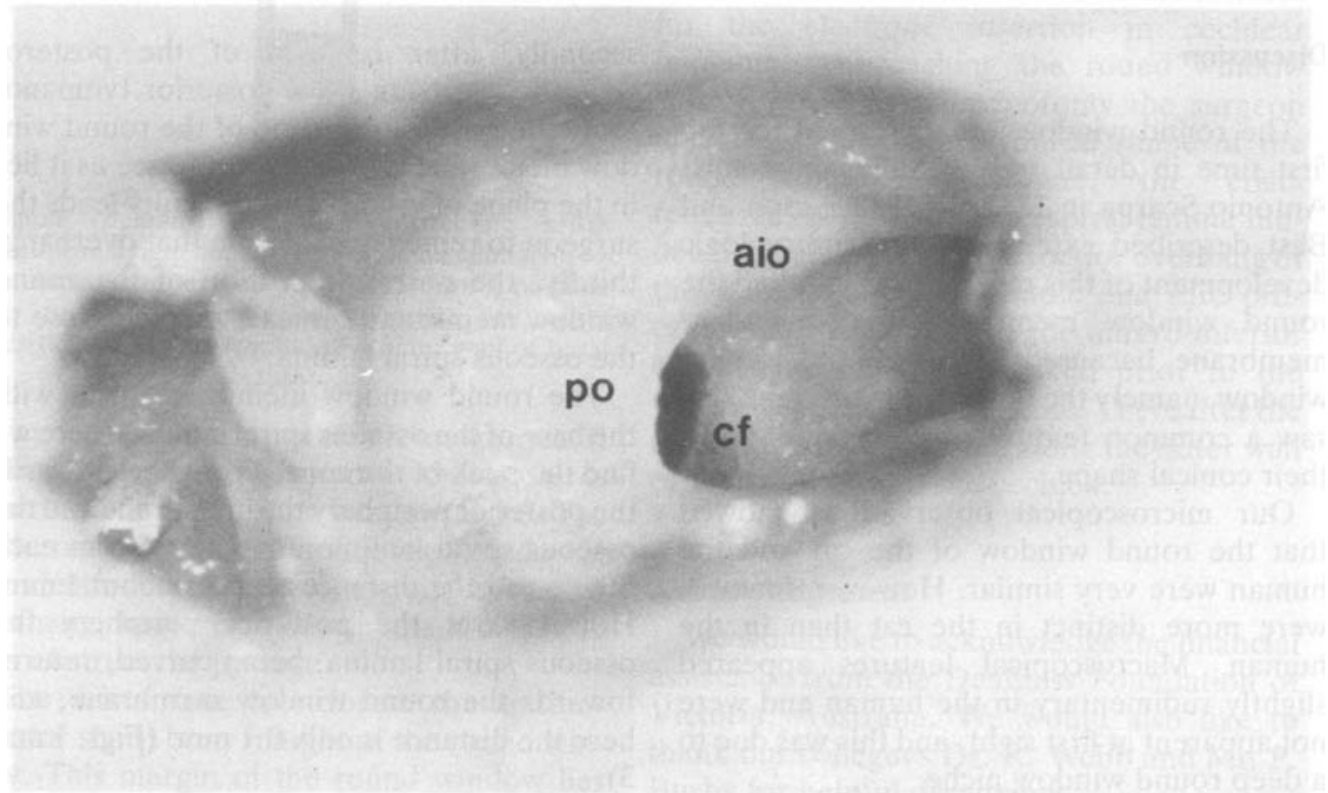

FIG. 2

View of the round window from a posterior tympanotomy in a right human temporal bone. The posterosuperior overhang impedes visibility of the posterior portion of the round window membrane. aio: anteroinferior overhang; po: postero-superior overhang; cf: crista fenestrae. 


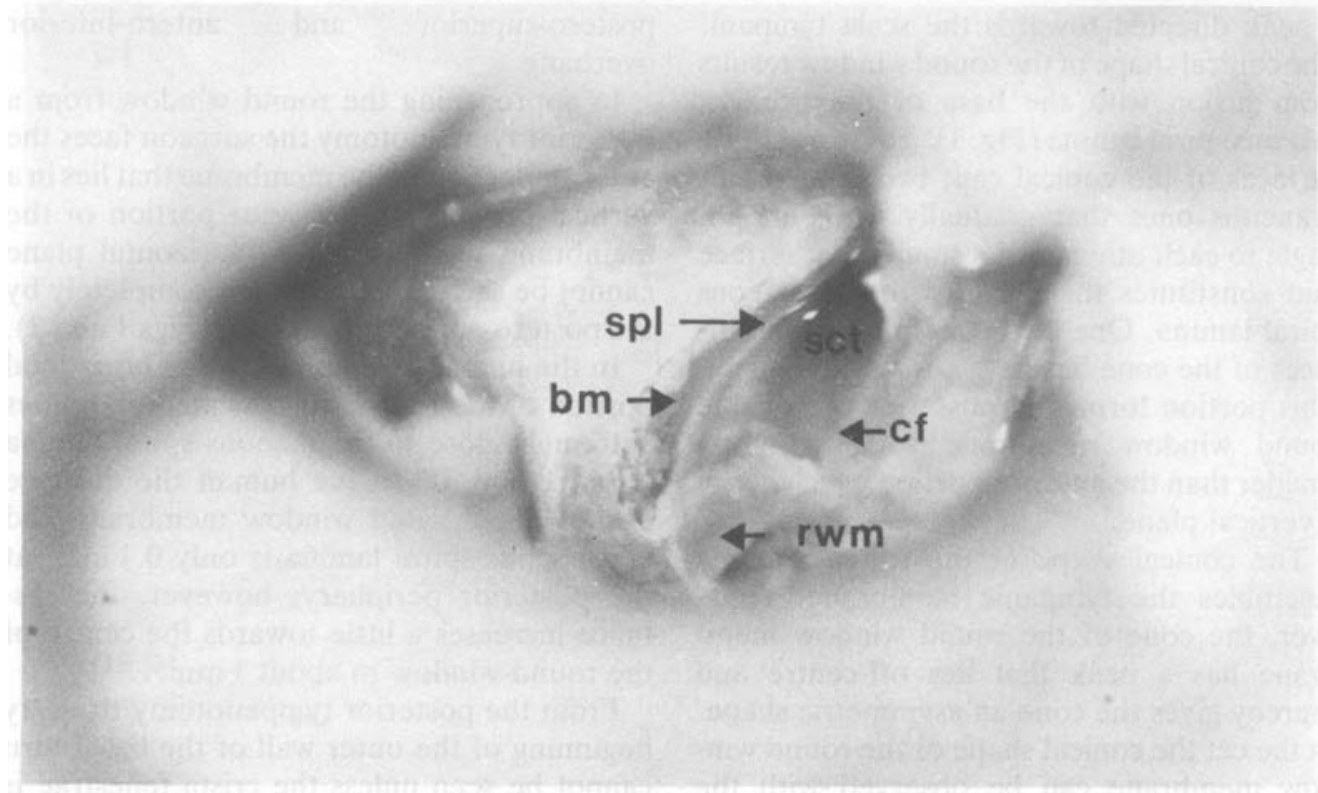

Fig. 3

The right round window in a human temporal bone as seen from an endaural approach demonstrating the close relationship between the osseous spiral lamina and the posterior portion of the round window membrane. For better demonstration the postero-superior and the antero-inferior overhang have been removed. bm: basiliar membrane; spl: spiral lamina; sct: scala tympani; rwm: found window membrane, cf: crista fenestrae.

\section{Discussion}

The round window was described for the first time in detail by the Italian anatomist Antonio Scarpa in 1772. In 1953 Anson and Bast described extensively the embryologic development of this region. Scarpa called the round window membrane the secondary membrane, because it resembled the primary window, namely the tympanic membrane. He saw a common feature of both windows in their conical shape.

Our microscopical observations showed that the round window of the cat and the human were very similar. However, features were more distinct in the cat than in the human. Macroscopical features appeared slightly rudimentary in the human and were not apparent at first sight, and this was due to a deep round window niche.

In the human there are three important characteristics of the round window niche: firstly, the postero-superior overhang is much smaller than the antero-inferior overhang; secondly, after removal of the posterosuperior overhang via a posterior tympanotomy the posterior portion of the round window membrane is still difficult to see as it lies in the plane of vision. This difficulty leads the surgeon to remove more from that overhang; thirdly, the posterior portion of the round window membrane comes extremely close to the osseous spiral lamina.

The round window membrane fuses with the base of the osseous spiral lamina where we find the peak of the cone. From here towards the posterior periphery the membrane and the osseous spiral lamina move away from each other, and the distance reaches about $1 \mathrm{~mm}$. However, at the posterior periphery the osseous spiral lamina, being curved, returns towards the round window membrane, and here the distance is only $0.1 \mathrm{~mm}$. (Figs. 1 and 3).

When the postero-superior overhang is removed the spiral lamina can be traumatised. While the surgeon uses a diamond burr he might not be aware of breaking the spiral lam- 


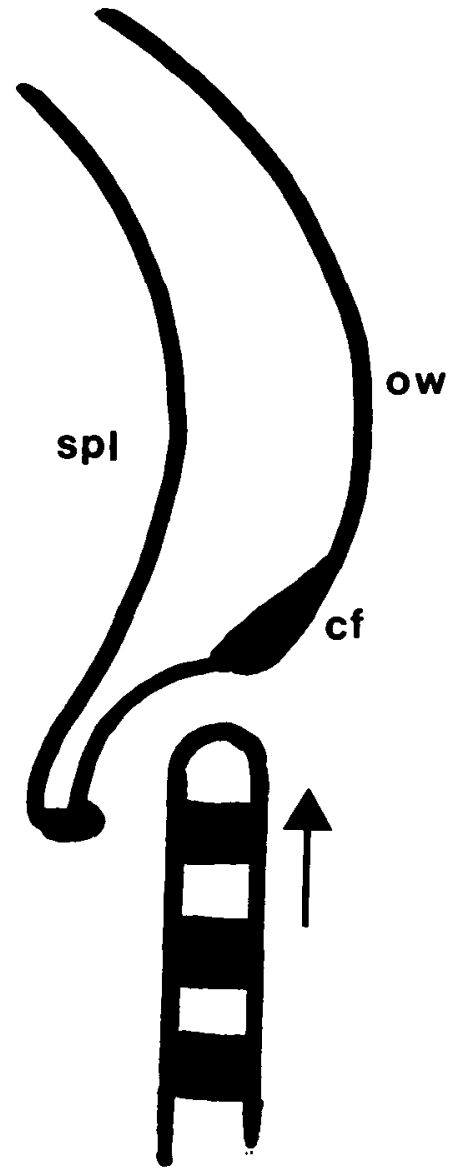

FIG. 4

Diagram demonstrating the obstructing crista fenestrae in a right human cochlea. A tangential insertion along the outer wall of the basal turn is possible after removal of the crista fenestrae. cf: crista fenestrae; spl: spiral lamina; ow: outer wall of basal turn.

ina underneath the intact elastic membrane. It is not necessary to remove the posterosuperior overhang of the window niche. The postero-superior overhang is not hindering the electrode insertion. The antero-inferior overhang, however, and the crista fenestrae may obstruct the passage.

The crista fenestrae forms the anteroinferior sharp bony margin of the round window. This margin of the round window lies closer to the modiolus and the osseous spiral lamina than the outer wall of the scala tympani in the basal turn of the cochlea. When the crista fenestrae is left in place only the medial portion of the scala tympani gives room for the electrode insertion. The narrow space between the crista fenestrae and the modiolus can cause friction, and the electrode cannot be led tangentially along the outer wall of the basal turn right from the start (Fig. 4).

A good view along the basal turn of the cochlea is achieved when the antero-inferior overhang and the crista fenestrae are removed. Only after the removal of the crista fenestrae can the whole width of the scala tympani in the basal turn be seen and this gives enough space for the electrode insertion.

It is important to preserve the osseous spiral lamina anatomically and thus prevent a reduction of the nerve fibre population. When attention is paid to the above particular features of the round window at surgery, damage to the osseous spiral lamina should not occur, and a deep electrode insertion will be possible.

\section{Conclusions}

The round window is a convenient access for the electrode insertion in cochlear implants. Approaching the round window from a posterior tympanotomy the surgeon must be aware of the conical shape of the round window membrane, the crista fenestrae, and the osseous spiral lamina hidden beneath the postero-superior overhang of the window niche. A simple and safe procedure is the removal of the antero-inferior overhang. This step is taken prior to the removal of the crista fenestrae. Thereafter the electrode can be inserted along the outer wall of the basal turn of the cochlea.

\section{Acknowledgement}

We would like to acknowledge the financial assistance from the Deafness Foundation of Victoria, Australia. We would also like to thank our collegues Dr. R. Webb and Mr. P. Busby for helpful discussions.

\section{References}

Anson, B. J. and Bast, T. H. (1953) The development 
of the otic capsule in the region of the cochlear fenestra. Annals of Otology Rhinology and Laryngology, 62: 1083-1116.

Banfai, P. (1978) Zugangswege fuer das Cochlear Implant. HNO, 26: 85-89.

Burian, K., Hochmair-Desoyer, I. J. and Hochmair, E. S. (1981) Hoeren ueber ein cochlea implantat. Archiv für ONK-Heilkunde, 231: 569-570.

Chouard, C. D. and Mac Leod, P. (1976) Implantation of multiple intracochlear electrodes for rehabilitation of total deafness; preliminary report. Laryngoscope, 86: 1743-1751.

Clark, G. M. (1975) A surgical approach for a cochlear implant. Journal of Laryngology and Otology, 89: 9-15.

Clark, G. M., Pyman, B. C. and Bailey, O. R. (1979) The surgery for multiple-electrode cochlear implantations. Journal of Laryngology and Otology, 93: 215-223.

House, W. F. and Urban, J. (1973) Long term results of electrode implantation and electronic stimulation of the cochlea in man. Annals of Otology, 82: 504517.

Kubik, S. (1984) In: Cochlear Implants in Clinical Use. Advances in Audiology Vol. 7 (Keidel, W. D. and Finkenzeller, P., eds.) pp. 108-118, Karger, S.
Michelson, R. P. (1971) Electrical stimulation of the human cochlea. Archives of Otolaryngology, 93: 317-323.

Michelson, R. P. and Schindler, R. A. (1981) Multichannel cochlear implant. Preliminary results in man. Laryngoscope, 91: 38-42.

Scarpa, A. (1772) Anatomical observations concerning the structure of the round window of the ear and the secondary tympanum. Translated by Sellers and Anson 1962. Archives of Otolaryngology, 75: $16-45$.

Simmons, F. B. (1966) Electrical stimulation of the auditory nerve in man. Archives of Otolaryngology, 84: 2-54.

Address for Reprints:

Burkhard K. H. Franz, M.D.,

Department of Otolaryngology,

University of Melbourne,

Parkville 3052,

Australia.

Address for Correspondence:

Graeme M. Clark, Ph.D., F.R.C.S.,

Department of Otolaryngology,

University of Melbourne,

Parkville 3052,

Australia. 


\section{University Library}

\section{- M M N E R VA A gateway to Melbourne's research publications}

Minerva Access is the Institutional Repository of The University of Melbourne

Author/s:

Franz, Burkhard K. H.;Clark, Graeme M.;Bloom, David M.

Title:

Surgical anatomy of the round window with special reference to cochlear implantation

Date:

1987

Citation:

Franz, B. K. H., Clark, G. M., \& Bloom, D. M. (1987). Surgical anatomy of the round window with special reference to cochlear implantation. Journal of Laryngology and Otology, 101(2), 97-102.

Persistent Link:

http://hdl.handle.net/11343/27250 